\title{
Proposal of an ISO/IEEE11073 Platform for Healthcare Telemonitoring: Plug-and-Play Solution with new Use Cases
}

\author{
M. Galarraga, Student Member IEEE, I. Martínez, L. Serrano, Senior Member IEEE, P. de Toledo, \\ J. Escayola, J. Fernández, S. Jiménez-Fernández, S. Led, M. Martínez-Espronceda, E.Viruete and J. García
}

\begin{abstract}
Remote patient monitoring in e-Health is everyday closer to be a mature technology / service. However, there is still a lack of development in areas such as standardization of the sensor's communication interface, integration into Electronic Healthcare Record systems or incorporation in ambient-intelligent scenarios. This work identifies a set of use cases involved in the personal monitoring scenario and highlights the related features and functionalities, as well as the integration and implementation difficulties found when these are to be implemented in a system based on the ISO/IEEE11073 (X73) standard. It is part of a cooperative research effort devoted to the development of an end-to-end standards-based telemonitoring solution. Standardization committees are working towards adapting the X73 standard to this emerging personal health devices market and use case identification is essential to direct these revisions.
\end{abstract}

\section{INTRODUCTION}

$\mathrm{E}$ -Health offers a wide range of solutions in patient telemonitoring where scenarios are built up with several device combinations for vital signal acquisition [1]. Personal and Body Area Network (PAN / BAN) are emerging as wireless communications advance. Nevertheless, these solutions are unpractical without the use of standardization [2]-[4].

ISO/IEEE11073 standards (X73) were designed to address Intensive Care Unit (ICU) scenarios [5]. Authors claim that X73 can be adapted to these personal telemonitoring [6], and analyze a set of Use Cases (UC) as a starting point to the identification of new requirements to contribute to standards advancement. This work is based on the know-how achieved in previous .development and evaluation efforts.

This research work has been partially supported by projects TSI2005-07068-C02-01 and TSI200404940-C02-01 from Ministerio de Educación y Ciencia (Spanish Government), and a personal grant to both M.Galarraga and M. MartinezEspronceda from Navarre Regional Government. I.Martínez, J.Escayola, J.Fernández, E.Viruete and J.García are with the Communications Technologies Group (GTC) - Aragon Institute for Engineering Research (I3A) - Univ. Zaragoza (UZ), Spain (e-mail: imr@unizar.es). M.Galarraga, L.Serrano, S.Led and M.Martínez-Espronceda are with the Electrical and Electronics Engineering Dept. - Public Univ. Navarre (UPN), Spain (e-mail: miguel.galarraga@unavarra.es). S. Jiménez-Fernández is with the Bioengineering and Telemedicine Research Centre (GBT) - Technical Univ. Madrid (UPM), Spain. P. de Toledo is with the Computer Science Department, Carlos III Madrid Univ. Spain. (e-mail: paula.detoledo @uc3m.es).
Unlike ICU scenarios, these emerging situations involve communications restrictions due to particular electronic features as low voltage-low power sensors and processors included in wearable Medical Devices (MD), or even wired or wireless technologies not yet supported by X73. This means that the communication protocols need to be lighter, avoiding long time communications and being efficient in terms of overhead, bandwidth and use of CPU [7]. With the existing technology this leads to the conclusion that the most of the system's intelligence can not be placed near to the MDs or sensors that are monitoring the patient. In this way, the new features of the e-Health scenarios are driving a deep review of the X73 standards, envisioning a new profile for Personal Health Devices (PHD) communications [8].

Use cases proposal and implementation is likely the best way to detect disagreements between the X73 standard and the needs in e-Health scenarios, as well as to foresee potential solutions. Identified needs are systems based on a standard protocol, where each module can be replaced by a similar standard-one, taking advantages of Plug-and-Play (P\&P) capabilities for a simple configuration. Data should flow seamlessly from a sensor in the patient's home to a hospital, interacting with the patient's Electronic Healthcare Record (EHR). In short, bringing up advantages in costs, interoperability, comfort, and system usability for patients.

Besides the use cases, this paper describes a prototype platform where these functionalities are implemented and which is compliant with X73 and other standards (EN13606 or HL7). It is prepared to include (with minor modifications) features not yet supported by $\mathrm{X} 73$, such as wireless communications, and it is ready to adopt the planned changes brought to X73 by the PHD profile. Currently, this work is yet in a laboratory phase. The research group has been developing an X73 implementation focused in wired MDs (blood pressure and pulse-oximeter with USB/RS-232 connections) for controlled home scenarios, and a prototype based on wireless MDs (scale, ECG and pulse-oximeter with Bluetooth/RFID technologies) for personal sensor networks. Roadmap to the final development involves porting the software to developed personal health devices.

Use cases are presented in Section II., while the X73 platform is described in Section III. New functionalities and improvements based on the Ambient Intelligence (AmI) paradigm are described in Section IV. Finally, conclusions of the work are presented in Section V. 


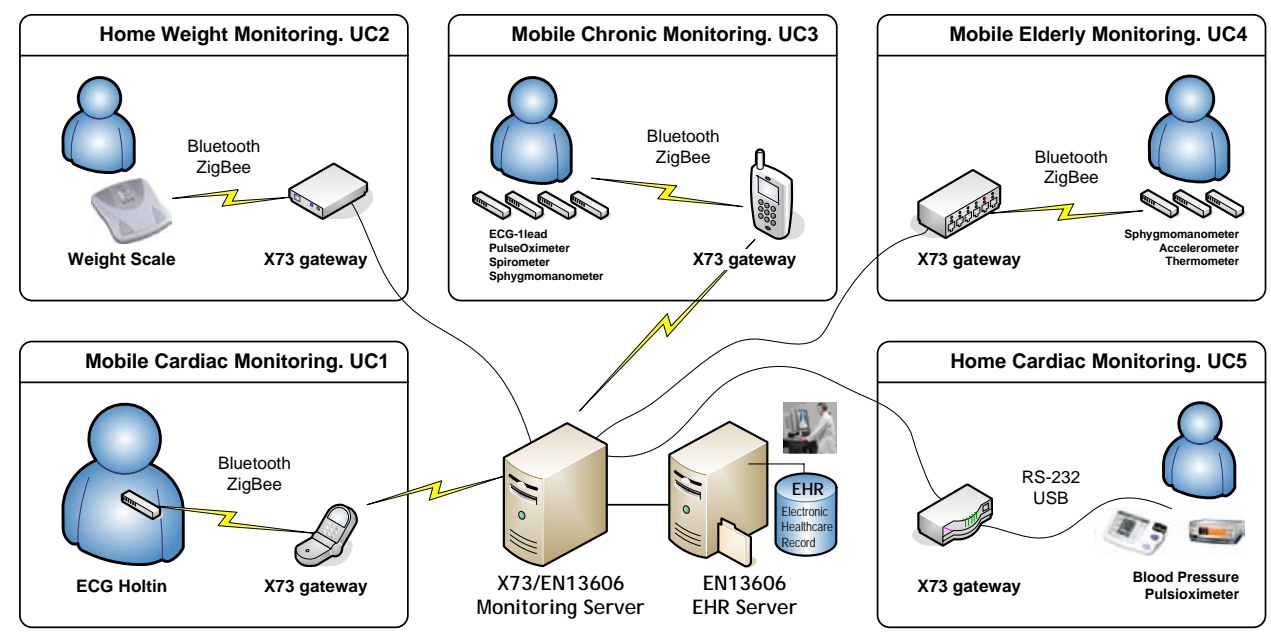

Fig. 1. UCs generic scheme

\section{II.PROPOSAL OF NEW USE CASES}

The proposed integrated solution that includes all these UCs is shown in Fig.1. The data from the different X73 MDs (according to each UC) is fetched in their X73 gateways, transmitted and managed by the X73/EN13606 Monitoring Server (MS), and stored in the EN13606 EHR server. The specific characteristics of each UC are following detailed:

\section{A. UC1 - Mobile cardiac monitoring}

A patient that suffers syncope or sporadic non perceptible symptoms of cardiovascular diseases could use the HOLTIN monitoring service [9]. The system is based on a native X73 wearable Holter device which controls the patient's ECG signal for long time periods in order to detect possible cardiac events. These events are transmitted, with Store-andForward (SF) scheme, to a X73-compatible mobile phone via Bluetooth and retransmitted to the MS by means of General Packet Radio Service (GPRS) technology, see Fig.1.

A front-end device located at the patient's chest performs both the acquisition of a modified ECG lead II (with a sampling rate of 200 samples/s) and the automatic detection of several cardiac events (tachycardia, bradycardia and asystolia) based on a QRS-detection algorithm. Moreover, a hand-triggered event is also possible if the patient notices he/she is having a specific symptom or suffers some syncope. Detected events are temporally stored in the device (up to $80 \mathrm{~min}$.) for a later transmission to the gateway.

Setting of specific HOLTIN features as gain, cardiac event threshold values, signal store time for both automatic or hand-triggered event detection and patient data, can be configured by health professionals based on patient pathology. Likewise alarms and warnings are run on both front-end and X73 gateway informing about power supply, limit of memory, number of events, etc.

\section{B. UC2 - Home weight monitoring.}

A healthcare professional prescribes this use case when he/she is concerned about the patient's weight (situation very common in cardiac arrest patients). The connectivity between the X73-compatible weigh scale and the X73gateway is achieved via Bluetooth or ZigBee, see Fig.1. Each time the patient follows the weighing protocol, her/his weight is transmitted to the X73-gateway and then to the MS via Internet. Once there, a specialist can access the MS to inspect the recorded data. The weigh scale is P\&P and the patient only needs controlling the battery status.

\section{UC3 - Chronic respiratory patient management}

A patient with a chronic respiratory condition (typically COPD [10]) self-monitors her/his status, usually once a day. Monitoring results are: spirometry (main values and flow volume curve), oxygen saturation (pulse-oximeter), and answers to a symptoms questionnaire. In case the patient has a co-morbidity, typically coronary disease or diabetes, monitoring may as well include ECG, non-invasive blood pressure, and weight (cardiac) or blood glucose levels (diabetes). The service is prescribed for chronic respiratory patients and it may be used during an exacerbation episode or for longer periods. The case manager, with technical staff support, provides the patient with a cellular phone (see Fig.1) prepared with the monitoring application, as well as the spirometer and pulse-oximeter. The information is transmitted to the MS once the different data have been gathered (immediately after), and patient is warned if the transmission is not ok. The X73-gateway takes care of retransmission if necessary. Transmission is SF and is not time critical, provided that it is sent within the same day. Case manager checks patient's status daily and receives alarms triggered by the system. The service is supervised by the technical staff from the service provider which takes care 
of the equipment. The gateway informs the patient if the monitoring MDs are not working properly (no batteries, device not connected or not working). Finally, warnings (SMS message, beeper call, email, web page) are issued if values are outside normal levels (personalized to the patient), if no data are received after a pre-established period, etc. Case manager or social worker may call the patient or pay a visit if needed.

\section{UC4 - Elderly patient follow-up.}

Similarly to UC3, the patient's vital signs are controlled once a day using several sensors. There are two main differences between both use cases: 1) a wearable 3D accelerometer (that records patient movements during all day) is included to detect falls and/or to obtain a summary of her/his level of activity. 2) The gateway uses a fixed internet access to transmit data to the MS, as in UC1. As depicted in Fig. 1, note that data transmission between the X73-gateway and each of the sensors is wireless.

\section{E. UC5 - Home cardiac monitoring.}

A single patient concerned with his heart health at home wants his cardiac parameters to be controlled by a cardiologist. The patient has a weigh scale, a blood pressure and a pulse and oxygen saturation measurement equipments installed at home using a fixed connection via RS-232 or USB, see Fig.1. Following the doctor's advice, he has to use the equipment several times a day.

A summary of the technical characteristics required for every UC is detailed in Table I. From this new proposal, the following section presents the X73 integration methodology for a P\&P solution.

TABLE I. TECHNICAL CHARACTERISTICS REQUIRED IN EVERY UC

\begin{tabular}{llllll}
\hline \multicolumn{1}{c}{ UC1 } & UC2 & UC3 & UC4 & UC5 \\
\hline Communications & & & & & \\
\hline X73-gateway possible inputs & & & & & \\
\hline Wireless (Bluetooth, Zigbee, ...) & $\checkmark$ & $\checkmark$ & $\checkmark$ & $\checkmark$ & \\
\hline Wired (RS-232/USB) & & & $\checkmark$ & $\checkmark$ & $\checkmark$ \\
\hline X73-gateway possible outputs & & & & & \\
\hline Wireless WAN & $\checkmark$ & & $\checkmark$ & & \\
\hline Wired WAN & & $\checkmark$ & & $\checkmark$ & $\checkmark$ \\
\hline Other features & & & & & \\
\hline X73 sensor adapter- $\mu$ controller & & & & $\checkmark$ & \\
\hline User warnings (SMS, e-mail, ...) & & & $\checkmark$ & & \\
\hline Battery status control & $\checkmark$ & $\checkmark$ & & $\checkmark$ & \\
\hline Faulty operation warnings & $\checkmark$ & $\checkmark$ & $\checkmark$ & $\checkmark$ & $\checkmark$ \\
\hline
\end{tabular}

\section{X73 INTEGRATION FOR P\&P SOLUTION}

The UCs previously presented aim to be implemented end-to-end, following the X73 standard [2] and integrated in a homogeneous telemonitoring X73 platform, see Fig.1. The X73 standard enables this integration in a modular way by defining each Virtual MD (VMD), its specific Domain Information Model (DIM), its working mode (baseline or polling), its communication technologies, etc. However, the existence of X73 MDs is strongly conditioned to the vendors and manufacturers. Currently there are a high percentage of MDs that not implement the X73 standard. Moreover, although the proposed UCs will permit to integrate them when they become available, it is also difficult to find MDs with an X73-compatible physical output: at this moment, only RS-232 and IrDA are included in X73 standard (but most extended interfaces are not allowed the: USB or Bluetooth).Thus, in this development, proprietary MDs have been used with X73-adapters for the UCs proposed. These adapters implement the X73 standard on one side, and proprietary interfaces that can only be modified by knowing the vendor programming codes on the other side. The X73 MDs and adapters included in the proposed solution (see Fig. 2) are described as follows:

- VMD1. Intelligent Holter (HOLTIN) is a low voltage-low power customized wearable device supplied with a Li-Po rechargeable battery. An 8-bit microcontroller performs the main tasks (acquisition of ECG signal, detection and storage of cardiac events) and a Bluetooth chip enables the communication with the mobile phone (X73-gateway in UC1) without adapter. However, a native X73 implementation on HOLTIN is a handicap that has to be addressed, due to the current X73 version does not include Bluetooth (although this will change in the PHD profile), and it also will require the standard to be lighter to avoid excessive power consumption.

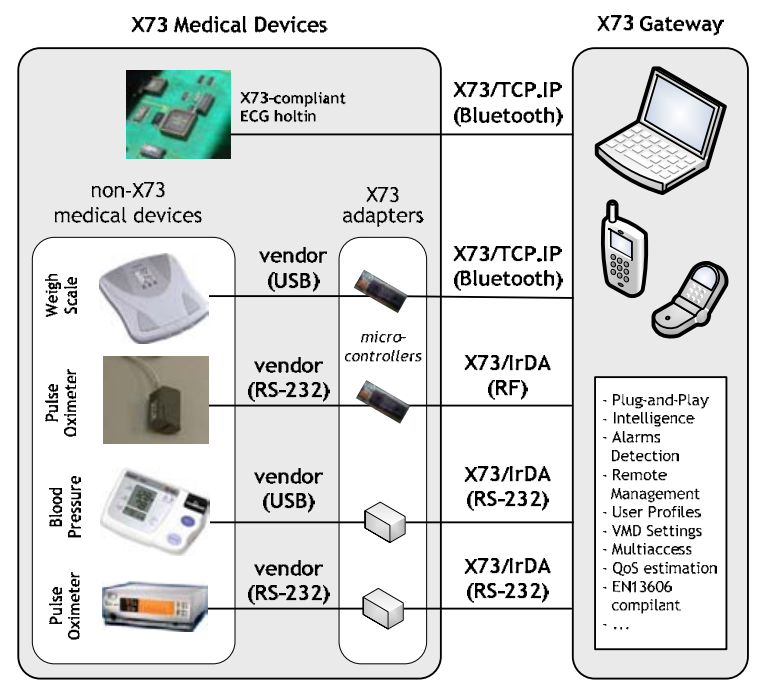

Fig. 2. MDs and X73-adapters considered in the solution 
- VMD2. The TEFAL PP1015B0 is a battery-powered commercial weigh scale provided with a Liquid Crystal Display (LCD). An ad-hoc adapter has been developed and integrated into the weigh scale for UC2. The adapter consists on a Peripheral Interface Controller (PIC), which taps data from the LCD via USO input interface, and manages the VMD communication in polling mode profile via a Bluetooth output module over a TCP/IP connection. The limited processing capacity and ultra-low power consumption required are the challenges to be addressed.

- VMD3. It is based on a MEDLAB EG00302 OEM pulse oximeter that measures blood oxygen saturation $\left(\mathrm{SpO}_{2}\right)$, heart rate and plethysmographic waveform, and provides communication via RS-232 input interface. To configure the X73 VMD, an attached microcontroller-based module supports a RadioFrequency (RF) output interface for UC3 and UC4. Default working mode is baseline (UC3), although a polling profile can be added if combined with an extra microcontroller (UC4).

- VMD4. The OMRON 705IT measures asynchronously the blood-pressure and the pulse rate, with a 28 acquisitions memory. It provides a USB connection (that requires a RS-232 adapter for its fully X73-compliance in UC5).

- VMD5. The DATEX-Ohmeda 3900 is a pulse-oximeter with a RS-232 serial-port output (the only wired interface included in X73 description) that measures $\mathrm{SpO}_{2}$, heart rate every 2s, and provides alarm support for UC5.

\section{AMBIENT INTELLIGENT FUNCTIONALITIES}

The proposed X73 integration platform contributes to a P\&P solution taking advantage of the X73 features in MDs interoperability. In order to improve the reliability and usability of this telecare platform, functionalities based on Ambient Intelligence paradigms have as well been considered in development. These AmI functionalities are:

- Quality of Service (QoS) analyzer module for evaluating the status of each internet access technology. As the number of MDs increases proportionally to the complexity of the monitoring scenario, the internet access must be properly managed, sharing the resources for each transmission's quality requirements. This module provides the possibility to adapt the transmission to the available bandwidth and required QoS.

- Alarm handling. X73 standard supports alarm reporting from MDs, so it is possible to trigger alarms on device and patient situations. Alarms can be reported to a healthcare professional when necessary, but could as well be handled by the patient at the gateway level. Intelligent alarm trigger on situations such as absence of patient response or detection of a worsening trend in vital signs is also possible.

- Personalized graphical and sensorial interface that helps guiding the patient in her/his monitoring procedure. Monitoring needs for a patient is gathered from the server that contains an up-to-date EHR file of the patient. This functionality is based in two configuration files: settings (with the VMD/X73-adapter proprietary technical characteristics, detailed in Table I) and profiles (with the user -patient, doctor- requirements, detailed in the UCs presented in Section II).

\section{V.CONCLUSION}

The work towards interoperable telemonitoring devices based on standards is mandatory to achieve mature e-Health solutions that are not dependent on a single vendor. It is interesting to approach this work from the perspective of different research groups, as interoperability problems arise faster than in proprietary developments. Ambient Intelligence platforms running on X73 gateways will also provide new functionalities with the aim to control the PAN status overall, interact with the patient through dedicated interfaces and report any hardware faulty operations or suspicious patient status to a remote control centre.. It is clear that the absence of medical device P\&P standards is an unacceptable barrier to innovation for safety and efficiency.

\section{ACKNOWLEDGMENT}

The authors wish to thank Mr. Melvin Reynolds, convenor of the CEN TC251 WGIV, for his key suggestions to this research. We also appreciate the contribution of the Research Groups of the Spanish Telemedicine Research Network.

\section{REFERENCES}

[1] R. Wooton, J. Craig, "Introduction to Telemedicine", ISBN-10: 1853156779, Rittenhouse Book Distributors, 2nd Ed, 2006.

[2] P. de Toledo, M. Galarraga, I. Martinez, L. Serrano, J. Fernández, F. del Pozo, "Towards e-Health Device Interoperability: The Spanish Experience in the Telemedicine Research Network", Int Conf IEEE Eng in Medicine and Biology Society, EMBS, pp. 3258-59, 2006.

[3] J. Yao, R. Schmitz, S. Warren, "A Wearable Point-of-Care System for Home Use That Incorporates Plug-and-Play and Wireless Standards". IEEE Trans Inf Technol Biomed, 9(3):363-371, 2005.

[4] S. Warren, J. Yao, R. Schmitz and J. Lebak, "Reconfigurable point-ofcare systems designed with interoperability standards," Annual International Conference of the IEEE Engineering in Medicine and Biology - Proceedings, 26(V):3270-3273, 2004.

[5] IEEE1073. Health informatics. Point-of-care Medical Device communication. Standard for Medical Device Communications Overview and Framework. http://www.ieee1073.org.

[6] M. Galarraga, L. Serrano, I. Martinez and P. de Toledo, "Standards for medical device communication: X73 PoC-MDC" Stud Health Technol Inform, vol. 121, pp. 242-256, 2006.

[7] J. Yao and S. Warren, "Applying the ISO/IEEE 11073 standards to wearable home health monitoring systems," Journal of Clinical Monitoring and Computing, vol. 19, pp. 427-436, 2005.

[8] Personal Health Devices PHD. IEEE Standards Association webpage: http://standards.ieee.org/. Last access: 21/06/07.

[9] S. Led, L. Serrano, M. Galarraga. "Intelligent Holter: a new wearable device for ECG", pp. 1527-1530 European Medical and Biological Engineering Conference, [HOLTIN project, in collaboration with Cardiology Dept. of Negrín Univ. Hospital, Canary Islands], 2005.

[10] P. De Toledo et al., "A telemedicine experience for chronic care in COPD", IEEE Trans Inf Techn Biomed, 2006. 